\title{
Structural Changes in Sodium Channel-Rich Nanodomains Contribute to the Progression of Atrial Fibrillation
}

\author{
Amara Greer-Short ${ }^{1}$, Thomas Hund ${ }^{1,2}$, Rengasayee Veeraraghavan ${ }^{1,2}$ \\ 1. Dorothy M. Davis Heart and Lung Research Institute, The Ohio State University, Columbus, USA. \\ 2. Department of Biomedical Engineering, The Ohio State University, Columbus, USA.
}

Atrial fibrillation (AF) is the most common type of arrhythmia; it affects $2-3 \%$ of the population, and elevates patients' risk of stroke and further cardiovascular disease [1]. Much of the healthcare challenge posed by AF derives from its progressive nature. Thus, there is an urgent need to uncover the mechanisms underlying AF progression, and identify methods of arresting it. Interestingly, AF patients have elevated levels of inflammatory cytokines such as vascular endothelial growth factor (VEGF) [2-5] and tumor necrosis factor $\alpha(\mathrm{TNF} \alpha)[6]$, which are associated with vascular dysfunction, a potential cause of myocardial edema. Previously, we have demonstrated proarrhythmic abnormalities in cardiac impulse propagation during myocardial edema secondary to disruption of intercalated disk (ID) nanodomain structure. Here, we investigated the hypothesis that increased vascular permeability promotes cardiac edema, and disrupts the molecular organization of the intercalated disc resulting in slowed conduction and elevated AF risk.

Super-resolution STochastic Optical Reconstruction Microscopy-based Relative Localization Analysis (STORM-RLA) identified two sub-populations of cardiac sodium channels ( $\left.\mathrm{Na}_{\mathrm{V}} 1.5\right)$ within the IDs of mouse atria, consistent with our previous observations in guinea pig ventricles [7]. Whereas one subpopulation, termed the perinexal pool, was localized within $200 \mathrm{~nm}$ of connexin43 (Cx43) gap junctions (GJs), the other, termed the plicate pool, co-distributed with $\mathrm{N}$-cadherin. In atria from untreated control mice, transmission electron microscopy (TEM) revealed tight intermembrane spacing within both perinexal $(9.1 \pm 1.5 \mathrm{~nm})$ and plicate $(26.2 \pm 0.9 \mathrm{~nm})$ nanodomains (fig 1, left). In contrast, atria from mouse hearts perfused with VEGF $(200 \mathrm{ng} / 1)$ for 20 minutes demonstrated significantly increased intermembrane distance within both perinexal $(48.4 \pm 6.5)$ and plicate $(90 \pm 4.9 \mathrm{~nm})$ nanodomains while other parts of the ID remained unaffected (fig 1, right). Atrial electrical activity was then assessed in untreated and VEGF-treated ex vivo mouse hearts by electrocardiography as well as optical mapping using the voltage-sensitive dye (di-4-ANEPPS). Both longitudinal and transverse conduction velocities were significantly lower in VEGF-treated hearts compared to untreated controls. Burst pacing (10s of pacing at decreasing cycle lengths of 50, 30, 25, and $20 \mathrm{~ms}$ ) failed to induce AF in all the control hearts tested. In contrast, burst pacing induced AF in 5 of 6 hearts tested.

These data suggest that disruption of ultrastructure within sodium channel-rich ID nanodomains, secondary to VEGF treatment, slows atrial impulse propagation and elevates the risk of AF. In summary, we provide the first evidence for a novel mechanism by which acute vascular leak induced by VEGF elevates AF risk and highlight sodium channel-rich ID nanodomains as potential targets for antiAF therapy.

References:

[1] Zoni-Berisso M et al, Clin Epidemiol 6 (2014), p. 213.

[2] Li J et al, Heart Rhythm 7 (2010), p. 438. 
[3] Ogi H et al, Circ J 74 (2010), p. 1815.

[4] Scridon A et al, Europace 14 (2012), p. 948.

[5] Seko Y et al, Jpn Heart J 41 (2000), p. 27.

[6] Kimura Tet al, Heart Lung Circ 23 (2014), p. 636.

[7] Veeraraghavan R and Gourdie R, Mol Biol Cell 10 (2016), p. 3583.
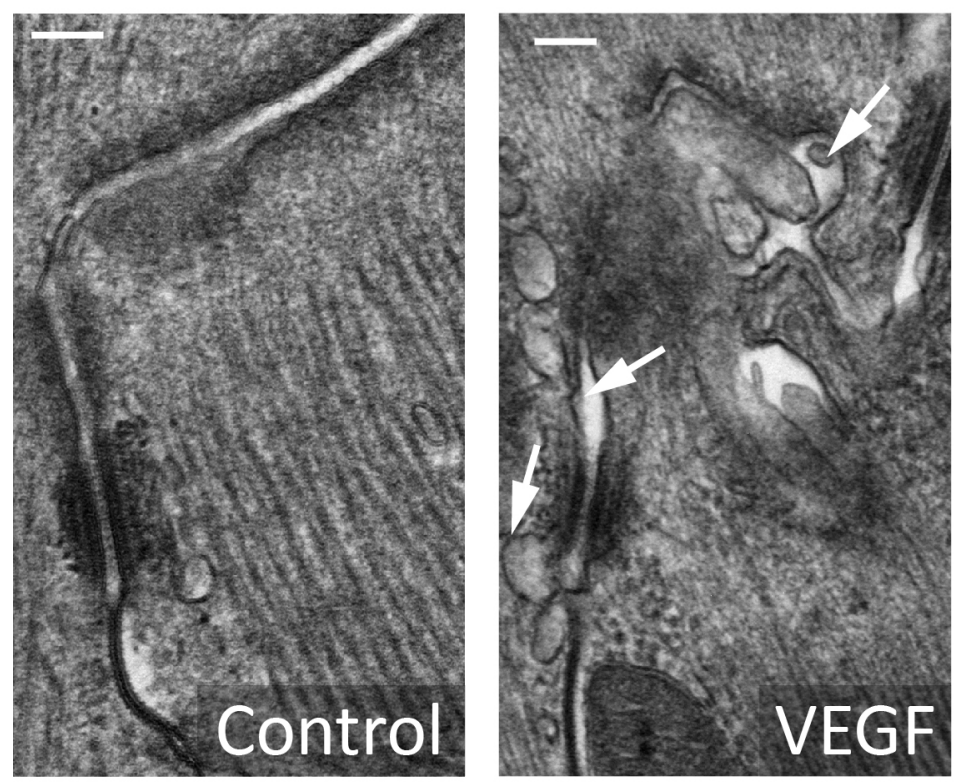

Figure 1: Transmission electron micrographs of atrial IDs from control and VEGF-treated hearts. White arrows highlight VEGF-induced changes in ultrastructure. Scale bars represent $100 \mathrm{~nm}$. 